\title{
Logistikprozesse für ein zentrales Motorenprüffeld - Planung und Bewertung von Systemalternativen des Materialflusses
}

\author{
Bianca Pellegrini, Herbert Sonntag
}

\section{Einleitung}

Ein funktionelles Materialflusssystem mit koordinierten Objektflüssen bildet die Basis für ein Unternehmen, effizient zu arbeiten und konkurrenzfähig zu bleiben. Zentrale Problemstellung dabei ist es, die einzelnen Teilbereiche aufeinander abzustimmen und in das Gesamtsystem zu integrieren. Prozessanalyse und Schnittstellendefinition sind die dazu erforderlichen Kernelemente. Sie schaffen die Voraussetzung dafuir, Optimierungspotenziale zu erkennen und entsprechende Maßnahmen zu planen. Die Tatsache, dass Logistik die methodische Entwicklung von solchen kompakten, systemuibergreifenden Konzepten anbietet, offenbart den hohen Stellenwert, den sie inzwischen vornehmlich im wirtschaftlichen Bereich einnimmt.

Ziel dieser Untersuchung war es, verschiedene Alternativen für das spezifische Materialflusssystem „,zentrales Heißpruiffeld“ in einem Motorenwerk der Automobilindustrie aufzuzeigen und durch die vergleichende Bewertung der erarbeiteten Varianten die Grundlage für eine Entscheidung seitens des Unternehmens zu schaffen. Am Ende steht ein Konzeptvorschlag, der die gestellten Anforderungen und Bedingungen bestmöglich erfüllt.

Vor dem Hintergrund von Materialdisposition und werkinternen Transport-, Umschlag-, Lagerungsprozessen (TUL) zählt die Bereitstellung der Prüfmotoren zum Aufgabenbereich der Produktionslogistik. Als Teilgebiet der Unternehmenslogistik umfasst Produktionslogistik die Planung, Steuerung und Überwachung der Material- und Informationsströme innerhalb eines Industriebetriebes. Sie stellt das Bindeglied zwischen Beschaffungs- und Distributionslogistik dar und organisiert die Güterbewegung von Roh-, Hilfs- und Betriebsstoffen, Werkzeugen, Kauf- und Ersatzteilen sowie Halbfertig- und Fertigfabrikaten zwischen Wareneingang und Distributionslager über die unterschiedlichen Produktionsstufen der Fertigung und Montage. Im bearbeiteten Fall sollen Materialien (Prüfmotoren) und zugehörige Informationen aus den verschiedenen Montagebereichen des Werkes zur zentralen Prüfstation und zurück fließen. Die Konzipierung der notwendigen Transport- und Zwischenlagerungsvorgänge muss dazu möglichst optimal auf die einzelnen Produktionsabläufe abgestimmt werden.

\section{Untersuchungsbereich und Systemschnittstellen}

Die Planung eines auf die neuen Anforderungen angepassten Material- und Informationsflusssystems inner- halb des Teilprojekts „Logistik“ wurde nochmals unterteilt in die Aufgabenpakete „Externe Logistik“ und „Interne Logistik“. Letztgenanntes Thema ist Gegenstand dieser Arbeit gewesen.

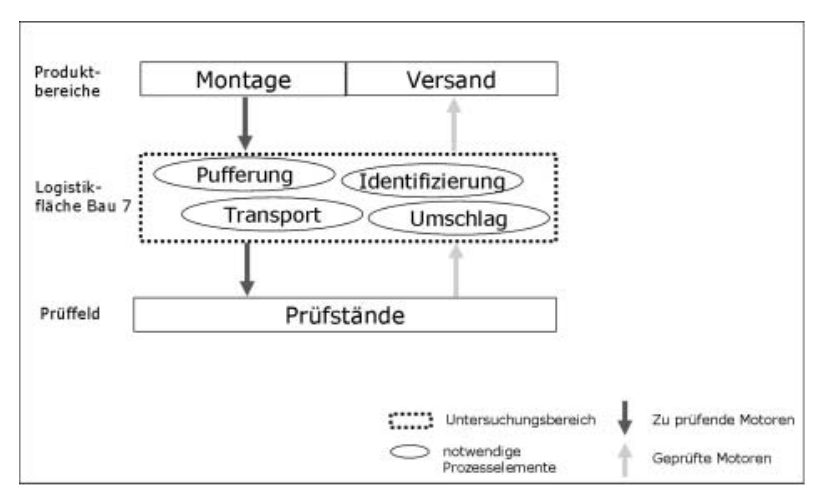

Abb. 1: Abgrenzung des Untersuchungsbereiches

Abbildung 1 verdeutlicht, welche Prozesse bei der Gestaltung des internen Logistikkonzeptes betrachtet werden sollten. Im Vordergrund standen dabei die TUL-Vorgänge zwischen der Anlieferung aus den Montagelinien und den Produktionsabläufen im Heißprüfzentrum. Die Planung der internen Prüffeldlogistik umfasste Entwicklung, Auswahl, Darstellung und Bewertung mehrerer Alternativen, die folgende Elemente abbilden:

- interner Materialfluss und Handling im Prüffeld: Anund Ablieferprozess für die Rüstplätze (Motorenzufuihrung, LT-Pufferung),

- Dimensionierung, Gestaltung und Organisation des Prüffeldpuffers,

- Transportabläufe und Fördertechnik,

- begleitender Informationsfluss im Prüffeld/Materialflusssteuerung.

Ein weiterer Schwerpunkt lag in der Abstimmung der Logistik mit der Layout- und Einrichtungsplanung.

\section{Ausgangssituation, Rahmenbedingungen und Planungsgrundlagen}

In der industriellen Motorenmontage der Automobilherstellung hat es sich inzwischen zum Standard herausgebildet, die Komponenten, Baugruppen und Fertigprodukte in unterschiedlichen Produktionsstufen mehreren Qualitätsprüfungen zu unterziehen. Unter verschiedenen Bedingungen werden diverse Parameter getestet, um die volle Funktionstüchtigkeit nachzuweisen und diese dem Kunden gegenüber gewährleisten zu können.

Im themenstellenden Unternehmen durchlaufen die montierten Motoren drei Funktionsprüfungen - einen 
sogenannten Kalt-Test (Endfunktionstest), einen Hot-Test (produktionsbegleitende Heißprüfung) und eine Guitesicherung (Giisi). Unmittelbar vor dem Einbau ins Fahrzeug werden im Rahmen der Heißprüfungen Funktionalität und Dichtheit der Motoren bei eigenem Antrieb uiberprüft. Unter stufenweise zunehmender Belastung lässt man den Prüfmotor warmlaufen, bis eine bestimmte Öltemperatur erreicht ist. Dabei werden alle für eine Produktionskontrolle wichtigen Motordaten gemessen, uiberwacht und dokumentiert. Zum Schluss der Warmlaufphase wird ein kurzer Leistungstest durchgefuihrt. Im Gegensatz zum Kalt-Test werden Heißprüfungen im Werk zentral abgewickelt und stichprobenhaft durchgefuihrt, wobei die Auswahl der Testmotoren nach einer internen Vorschrift erfolgt. Für die Prüfquote entscheidend sind Produktionsalter der Variante, Qualitätsergebnisse und Kalt-Testrate.

Alle Motortypen müssen aus den verschiedenen Produktionsstätten dem Prüffeld zugestellt und von diesem wieder abgeholt werden. Die Quellen und Senken der Prüfmotoren liegen im Werk verstreut, räumlich weit voneinander entfernt. Die Abwicklung des Prüfmotorentransportes zwischen dem Prüffeld und den Kundenabteilungen wurde im Umfeld der „Externen Logistik“ untersucht und erfolgt im Rahmen eines Ringroutenverkehrs. Ein LKW-Anhänger befördert Motoren aus den verschiedenen Produktionsstätten regelmäßig und gesammelt auf einer festen Route zur zentralen Logistikfläche im Prüffeld.

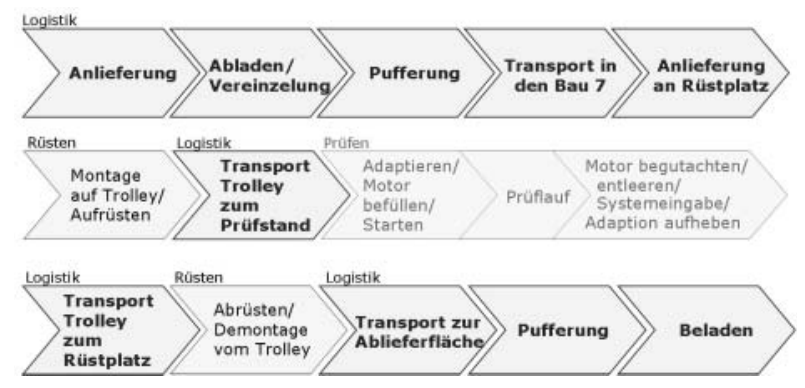

Abb. 2: SOLL-Prozess

In Abbildung 2 ist die Prozesskette des geplanten Ablaufs im Heißprüifzentrum dargestellt. Der von der „Externen Logistik“ empfohlene Routenverlauf mit einem ca. zweistuindigen Umlauf ist die vorgebende Einflussgröße bei der Dimensionierung des Prüffeldpuffers. Um den arbeitstäglichen Prüfumfang gleichmäßig zu verteilen und die Kapazität der Prüfstände optimal auszunutzen, sollen 12 Motoren pro Stunde bereitgestellt werden. Erfolgt die An- und Ablieferung alle zwei Stunden, sind demzufolge je 24 Ladeeinheiten mit einer Ladung umzuschlagen.

Aufgrund der beschränkten Platzverhältnisse soll kein Sicherheitsbestand im Prüffeldpuffer vorgehalten werden. Allerdings werden zum Ausgleich der Zeitversetzungen zwischen Anlieferungs- und Prüfzyklus je drei zusätzliche Stellplätze (Redundanz) eingeplant. Aus diesen Angaben resultiert die Bemessung der Stellplätze im Puffer: Eingang: 24 LE +3 LE Redundanz

Ausgang: 24 LE +3 LE Redundanz

Leergut: $10 \mathrm{LE}$
Im „Eingang-Puffer“ befinden sich die angelieferten, zu pruifenden Motoren; der „Ausgang“ (Versandpuffer) puffert die bereits geprüften Motoren bis zum Rücktransport in die jeweiligen Produktionsbereiche. Die Leergutplätze sind für leere Ladungsträger, die während der Prüfprozedur nicht am Rüstplatz verbleiben können und zwischengelagert werden müssen. Dies betrifft insbesondere Guitesicherungs- und Nacharbeitsmotoren. Der Leergutpuffer ist in Nähe des Rüstbereichs abzubilden und wird in Form von Bodenlagerung oder eines einfachen Fachbodenregals realisiert. Wie groß die Fläche des Puffers sein wird, ist vom zu wählenden System abhängig. Hier spielen Faktoren wie Abmessungen der technischen Einrichtungen, Anzahl und Größe der benötigten Verkehrs- und Bedienwege, Sicherheitsabstände usw. eine Rolle.

Voraussetzung für die Prozesssicherheit des Pufferlagers ist die physische und optische Trennung zwischen Eingang und Ausgang. Innerhalb des Eingangspuffers ist eine chaotische Lagerung möglich, da mit Hilfe der Transponder eine eindeutige Zuordnung von Motornummer und Lagerplatz gewährleistet wird. Beim Lagerausgang ist eine feste Lagerplatzvergabe zu empfehlen, damit die Lagerguiter leichter zu unterscheiden sind und für die Beladung des LKW-Anhängers eine Vorsortierung entsprechend der zu beliefernden Abteilungen erfolgen kann. Die chaotische Lagerung erfordert, dass die Auslegung der Materialflusstechnik anhand der Abmessungen der größten Ladeeinheit erfolgt. Auf diese Weise kann jeder Stellplatz jede Ladeeinheit aufnehmen.

Eine große Herausforderung fuir die Logistik bestand in der Vielfalt der zu handhabenden Ladeeinheiten. Eine Ladeeinheit setzt sich jeweils aus Motor und Ladehilfsmittel (Motorladungsträger) zusammen. Insbesondere die heterogenen Eigenschaften der LT traten bei der Auswahl der Materialflusstechnik häufig als restriktiver Faktor auf. Als Prämisse müssen die Prüfmotoren außerdem im selben LT ausgeliefert werden, in dem sie dem Prüffeld bereitgestellt wurden.

\section{Varianten für die Materialflussprozesse}

\subsection{Variante 1 „Schwerlastregal“}

Als Lagermittel wurde ein Fachbodenregalsystem gewählt, das räumlich zwischen LKW-Anhänger-Stellplatz und Prüfstandsgebäude gelegen ist und beidseitig bedienbar ist. Es werden konventionelle Schwerlastregale mit einer Mindest-Tragfähigkeit von $400 \mathrm{~kg}$ pro Stellplatz verwendet. Holzböden garantieren, dass die verschiedenen Größen der Lagergüiter aufgenommen werden können. Die unterste Ebene wird in Form von Bodenlagerung, also ohne Regalböden, genutzt. Jedes Regalfach im Puffereingang ist mit einem Transponder ausgestattet, der die Stellplatz-ID speichert.

\section{Prozessablauf}

Angelieferte Ladeeinheiten werden von Norden mit einem Stapler in das Regal eingelagert, auf der Südseite von einem Elektro-Deichselstapler entnommen und 

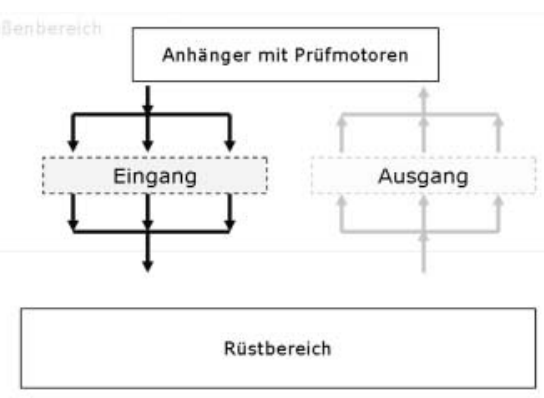

$\downarrow$ zu pratende LE 1 geprufte LE

Abb. 3: Prinzipskizze der Regalbestückung

weiter zum Rüstbereich befördert. Für den Puffer-Ausgang ist der Ablauf umgekehrt. Somit wird die Kreuzung der verschiedenen Materialflüsse vermieden.

\section{Systemkosten}

In Tabelle 1 sind die Gesamtkosten für Variante 1 in einzelne Kostenblöcke aufgeschluisselt. Auffallend sind die äußerst geringen Investitionen.

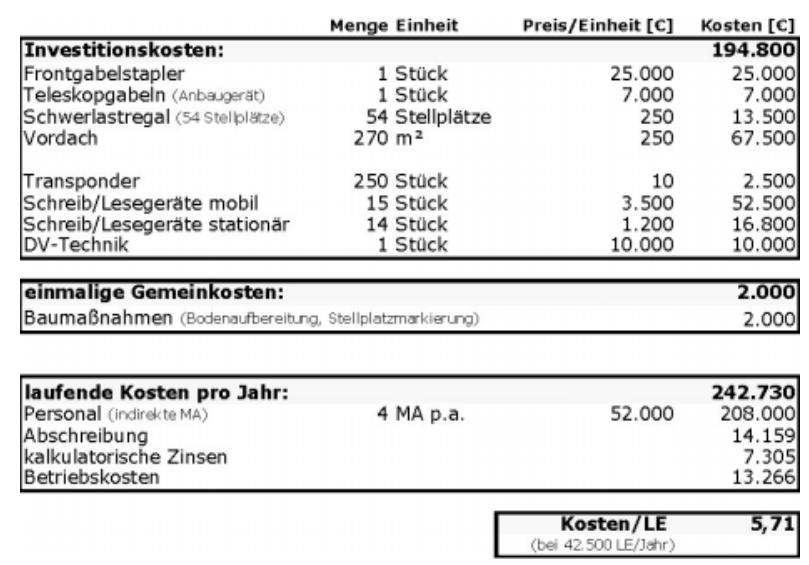

Tab. 1: Kostenkalkulation für die Variante 1

\section{Prozessbeurteilung}

Diese Variante zeichnet sich durch einen souveränen Prozess aus, der mit kleinstmöglichen Anschaffungskosten realisierbar ist. Vorteile sind:

- einfacher, sicherer Prozess,

- hohe Transparenz,

- hohe Flexibilität,

- geringe Investitionen,

- keine Baumaßnahmen am Gebäude und

- geringer Flächenverbrauch.

Nachteile:

- häufiges Handling $=>$ dadurch hohe Prozesskosten

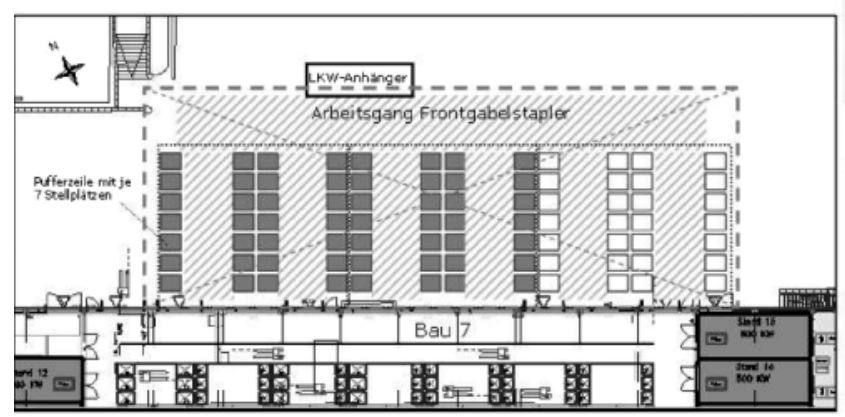

\subsection{Variante 2 „Fahrgestelle“}

Bei dieser Alternative werden die Ladeeinheiten per Stapler direkt vom Anhänger auf speziellen, fahrbaren Gestellen (Wagen) abgesetzt. Für die gesamte Zeit im Prüffeld verbleiben die Ladungsträger auf diesen Wagen, auch während der Motor den Ladungsträger zum Rüsten und Prüfen verlässt. Die Fahrgestelle haben eine Holzbodenplatte mit einer Ladefläche von 1010 x 1210 mm, eine Ladehöhe von $280 \mathrm{~mm}$ und eine maximale Tragfàhigkeit von $500 \mathrm{~kg}$. Zur leichteren Handhabung sind sie mit zwei lenkbaren und zwei fixen großen Rädern sowie einer Bremse ausgestattet und werden beim Be- und Entladen zusätzlich durch eine Spurfuihrungsschiene am Fußboden fixiert und ausgerichtet. Transponder-Tags, die auf den Boden aufgeschraubt sind, ermöglichen die Stellplatzzuordnung im Eingangspuffer. Da der Zugriff auf jede Ladeeinheit zu jeder Zeit möglich sein muss, werden die Wagen in Zeilenform angeordnet. Stapler und Fahrgestelle, die zwischen Puffer und Rüstbereich verkehren, nutzen im Puffer die gleichen Bedienwege. Das Personal muss entsprechend der erhöhten Unfallgefahr vorsichtig und rücksichtsvoll arbeiten.

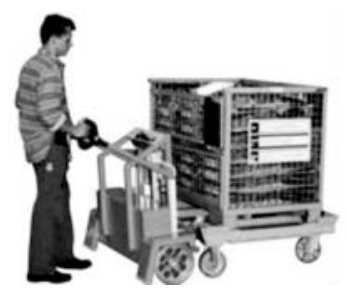

Abb. 4: Anwendungsbeispiel und Funktionsweise einer Andockstation

\section{Prozessablauf}

Die Bestuickung und Entleerung der Pufferwagen übernimmt ein Frontgabelstapler. Da die Platzierung der Ladeeinheiten auf den Fahrgestellen für einen Stapler komplizierter ist als z. B. die Einlagerung in einem feststehenden Regal, verlängern sich die Be- und Entladezeiten. Der Transport zwischen der vorgesehenen Pufferfläche und den Rüstplätzen erfolgt mit Hilfe der Fahrgestelle, indem ein Mitarbeiter sie verschiebt. Dabei minimiert eine spezielle Rahmen-Andockstation (Abb. 4) die erhöhte Unfallgefahr und die starke körperliche Belastung des Bedienpersonals beim Bewegen der schweren,

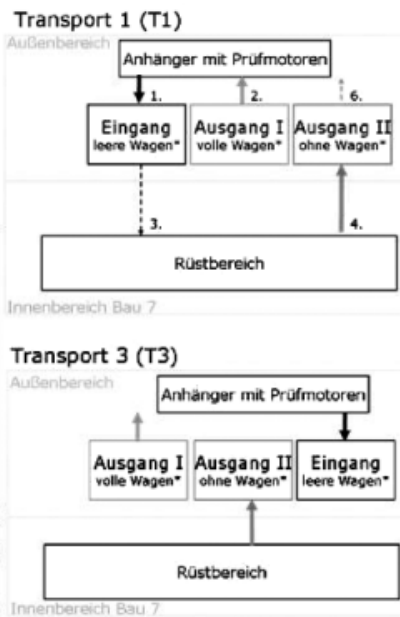

Abb. 5: Layout und Prinzipskizze Variante 2 
unhandlichen Fahrgestelle. Die Station wird per Knopfdruck mit einer speziellen Technik automatisch an den Fahrgestellrahmen ,angedockt“ und ebenso schnell wieder entkoppelt. Der Einsatz solcher Andockstationen erleichtert und beschleunigt die Handhabung der Wagen, besonders den Transport zwischen Puffer und Rüstbereich. Mit der Anschaffung solcher Stationen ist auf der anderen Seite ein entsprechender Mehraufwand an Investitionen verbunden.

Beim Platzieren der Transportgestelle am Rüstplatz muss die Bremse festgestellt werden. Wagen mit Leergut von Giisi- und Nacharbeits-Motoren werden, um Platz zu schaffen, auf eine gekennzeichnete Fläche am Rand des Gebäudes geschoben. Wenn die Wagen auf die Außenpufferfläche geschoben werden, ist darauf zu achten, dass sie in die Bodenschiene eingeführt werden. Außerdem sollten sie innerhalb der Stellplatzmarkierung abgestellt werden, damit sie andere Plätze nicht blockieren und die Zuordnung uiber den Transponder eindeutig ist. Um eine relativ hohe Prozesssicherheit gewährleisten zu können, ist die Ordnung des Puffers ist mit einem hohen Steuerungsaufwand verbunden ist. Die Ursache hierfür liegt darin, dass der Verbrauch im Puffer nicht zeitsynchron zur Motoren-Anlieferung getaktet ist.

\section{Systemkosten}

Im Vergleich zu anderen Varianten zeigen sich bei den Fahrgestellen vor allem die Gemeinkosten für bauliche Veränderungen und der Investaufwand für das relativ große Vordach dominant. (Tabelle 2)

\begin{tabular}{|c|c|c|c|}
\hline & Menge Einheit & Preis/Einheit [c] & Kosten [C] \\
\hline Investitionskosten: & & & 301.300 \\
\hline Frontgabelstapler & 1 Stück & 25.000 & 25.000 \\
\hline Teleskopga beln (Anbaugesat) & 1 Stück & 7.000 & 7.000 \\
\hline Fahrgestelle & 100 Stück & 160 & 16.000 \\
\hline Spurführung & $65 \mathrm{~m}$ & 100 & 6.500 \\
\hline Andockstationen & 3 Stück & 7.500 & 22.500 \\
\hline Vordach & $570 \mathrm{~m}^{2}$ & 250 & 142.500 \\
\hline Transponder & 250 Stück & 10 & 2.500 \\
\hline Schreib/Lesegeräte mobil & 15 Stück & 3.500 & 52.500 \\
\hline Schreib/Lesegerăte stationär & 14 Stück & 1.200 & 16.800 \\
\hline DV-Technik & 1 Stück & 10.000 & 10.000 \\
\hline \multicolumn{3}{|l|}{ einm alige Gemein kosten: } & 15.000 \\
\hline \multicolumn{4}{|c|}{ Baumaßnahmen } \\
\hline laufende Kosten pro Jahr: & & & 262.979 \\
\hline Personal (indirekte MA) & 4 MA p.a. & 52.000 & 208.000 \\
\hline Abschreibung & & & 23.162 \\
\hline kalkulatorische Zinsen & & & 11.299 \\
\hline Betriebskosten & & & 20.519 \\
\hline & & $\begin{array}{l}\text { Kosten/LE } \\
\text { (bei } 42.500 \text { LE/Jahr) }\end{array}$ & 6,19 \\
\hline
\end{tabular}

Tab. 2: Kostenkalkulation für die Variante 2

\section{Prozessbeurteilung}

Vorteile:

- keine weiteren Umbauarbeiten am Gebäude notwendig

- äußerst flexible Handhabung im Prüffeld

- keine weiteren Umschlagvorgänge für Leergut im Ruistbereich

Nachteile:

- schwieriges Handling $=>$ längere Be- und Entladezeiten

- Dreckverschleppung

- hoher Flächenverbrauch, lange Fußwege für Logistikmitarbeiter
- erhöhte Unfallgefahr

- Organisation des Puffers schwierig $=>$ eingeschränkte Transparenz und Prozesssicherheit

\subsection{Variante 3 „Staukettenförderer“}

Ein Tragkettenförderer mit Rollenketten als Trag- und Zugorgan soll bei dieser Variante eine dynamische Lagerung abbilden. Das System ist mit zwei angetriebenen, dreistrangigen Stauförderstrecken ausgestattet. Eine Förderzeile ist als Eingangspuffer zu belegen. Sie befindet sich an der nördlichen Innenwand des Gebäudes. Die andere Förderstrecke dient als Ausgangspuffer und ist dem Gebäude außen vorgelagert. Die Kettenförderer sind als eine Art Durchlaufsystem gestaltet, bei dem an einer bestimmten Stelle die Ladeeinheiten aufgegeben und von dort bis zum letzten freien Stellplatz befördert werden. An den Aufgabepunkten befinden sich Wandöffnungen nach außen. Diese sind mit einer Schleuse in Form von Schnelllauftoren verschlossen. Statt am Stapler ist das stationäre Schreib-/Lesegerät zur Wareneingangserfassung am ersten Stellplatz der Eingangspufferzeile angebracht. Für die Stellplatzzuordnung ist es bei dieser Variante nicht notwendig, jeden Platz mit einem Transponder auszustatten.

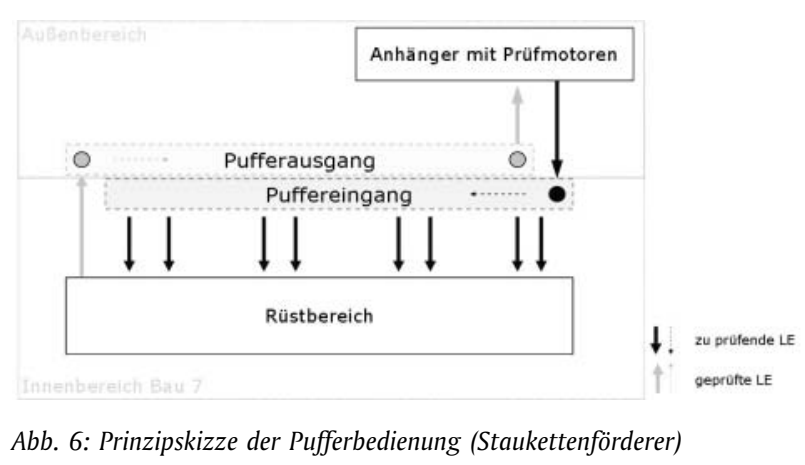

\section{Prozessablauf}

Beim Puffereingang werden die Ladeeinheiten mit einem Stapler von außen durch eine Schleuse nach innen auf den letzten Stellplatz gesetzt. Jeder Stellplatz ist durch eine Sperre vereinzelt, sodass zu jeder Zeit auf alle Ladeeinheiten zugegriffen werden kann. Die Bedienung der Förderzeilen erfolgt innerhalb des Gebäudes mit einem Elektro-Deichselstapler. Wenn eine Ladeeinheit aus der Reihe im Puffereingang entnommen wird, werden die Sperren der nachfolgenden Plätze in der Zeile gelöst und die Ladeeinheiten rücken automatisch auf. Für den Ausgangspuffer ist der Ablauf analog. An einer Übergabestelle werden die geprïften Ladeeinheiten durch eine Schleuse nach außen geleitet. Dort werden sie auf dem Kettenförderer bis zum letzten freien Platz weitertransportiert. Aufgrund des hohen Staudrucks, der entstehen würde, wenn mehrere Ladeeinheiten aufeinander geschoben werden, kann auch hierbei nicht auf eine Vereinzelung jedes Stellplatzes verzichtet werden.

\section{Systemkosten}

Tabelle 3 beinhaltet die Kostenaufteilung der Variante „Staukettenförderer“. Es fallen vor allem die hohen Investitionen für das spezielle Fördersystem ins Gewicht. 


\begin{tabular}{|c|c|c|c|}
\hline & Menge Einheit & Preis/Einheit [C] & Kosten $[\mathrm{C}]$ \\
\hline Investitionskosten: & & & 430.800 \\
\hline Frontgabelstapler & 1 Stück & 25.000 & 25.000 \\
\hline Teleskopgabeln (Anbougerat) & 1 Stück & 7.000 & 7.000 \\
\hline Staukettenförderer & 2 Stück & 160.000 & 320.000 \\
\hline Vordach & $50 \mathrm{~m}^{2}$ & 250 & 12.500 \\
\hline Schleuse & 2 Stück & 5.000 & 10.000 \\
\hline Transponder & 200 Stück & 10 & 2.000 \\
\hline Schreib/Lesegeräte mobil & 15 Stück & 3.500 & 52.500 \\
\hline Schreib/Lesegeräte stationär & 14 Stück & 1.200 & 16.800 \\
\hline DV-Technik & 1 Stück & 10.000 & 10.000 \\
\hline \multicolumn{3}{|l|}{ ein malige Gemeinkosten: } & 3.000 \\
\hline \multicolumn{3}{|c|}{ Bauma 3 nahmen (Wenddurchbruche, Stell platemarkerung) } & 3.000 \\
\hline \multirow{6}{*}{\multicolumn{2}{|c|}{$\begin{array}{l}\text { laufende Kosten pro Jahr: } \\
\text { Personal (indrekte MA) } \\
\text { Abschreibung } \\
\text { kalkulatorische Zinsen } \\
\text { Betriebskosten } \\
\end{array}$}} & & 287.118 \\
\hline & & 52.000 & 208.000 \\
\hline & & & 33.626 \\
\hline & & & 16.155 \\
\hline & & & 29.337 \\
\hline & & $\begin{array}{c}\text { Kosten/LE } \\
\text { (bet } 42,500 \text { LE/Dahr) }\end{array}$ & 6,76 \\
\hline
\end{tabular}

Tab. 3: Kostenkalkulation für die Variante 3

Einsparungen bei den Kosten für Überdachung und Transponder können diese Unterschiede bei weitem nicht ausgleichen.

\section{Prozessbeurteilung}

Die Vorzuige dieser Alternative, nämlich geringer Flächenverbrauch und Teilautomatisierung, können die schlechte Wirtschaftlichkeit nicht kompensieren.

Vorteile:

- im Gebäude integrierbar P geringer Flächenverbrauch

- definierte Übergabepunkte $=>$ kurze Staplerwege, kleines Dach

- vereinfachte Transponder-Erfassung mit Lagerplatzzuordnung

Nachteile:

- hohe Investitionen durch Speziallösung

- unterschiedliche LHM problematisch

- Umbauten am Gebäude erforderlich

- häufiges Handling

- unflexibel

\subsection{Variante 4 „LKW-Anhänger“}

Gerade bei Zwischenlagerungsvorgängen im Fertigungsprozess kann es durchaus sinnvoll sein, ein Transportmittel gleichzeitig als Puffer zu nutzen. Neben finanziellen
Einsparungen für zusätzliche Lagertechnik (wie Regale) können häufig Reduzierungen hinsichtlich Flächenverbrauch und Handlingsaufwand erzielt werden. Grundsätzlich erscheint es also angebracht, das Prüffeld direkt aus dem anliefernden LKW-Anhänger mit Prüfmotoren zu versorgen, ohne dass weitere Umschlagstufen dazwischengeschaltet sind. Auf diese Weise entfallen Handhabungsvorgänge für die Bestuickung und Entleerung des Puffers, die in diesem Fall mit der Be- und Entladung des Anhängers gleichzusetzen sind. Als weitere Konsequenz daraus verkürzt sich die Umlaufzeit des Routenverkehrs. Es wurde bereits angedeutet, dass dann alle anderthalb Stunden angeliefert werden kann. Folglich verringern sich die Stuickzahlen pro Anlieferung und die im Puffer vorgehaltenen Stellplätze.

\section{Beschreibung der Systemtechnik}

Vier LKW-Anhänger und ein Gabelstapler sind die notwendigen Systembestandteile. Der als Planungsprämisse geforderte Direktzugriff auf alle Ladeeinheiten im Puffereingang kann bei dieser Systemalternative nur unter bestimmten Bedingungen realisiert werden:

(1)Es muss eine beidseitige Entladung der Anhänger möglich sein.

(2)Auf dem LKW-Anhänger dürfen maximal zwei Ladeeinheiten hintereinander stehen.

(3)Es sind spezielle Anhänger erforderlich: Sie verfügen uiber zwei separate Ebenen, die durch eine Zwischenplattform getrennt sind. Ein weiterer Vorteil, der daraus entsteht, ist die Vermeidung von gelegentlichen Umstapelungen auf der Route sowie die Garantie, dass auch nicht stapelbare Ladeeinheiten übereinander gelagert werden können. Allerdings steigen die Investitionen in die Systemtechnik.

Um auch im Anhängerpuffer eine eindeutige Stellplatzzuordnung zu ermöglichen, ist pro Platz ein Transponder aufgeschraubt. Ähnlich wie bei den Fahrgestellen in Variante 2 ist die Voraussetzung, dass die einzelnen Stellplätze voneinander abgegrenzt sind und sich jeweils nur eine Ladeeinheit im Erfassungsbereich des StellplatzTransponders befindet. Die Abgrenzung wird in Form von L-Profilen erfolgen, die auf den Boden der Anhängerebenen montiert werden.

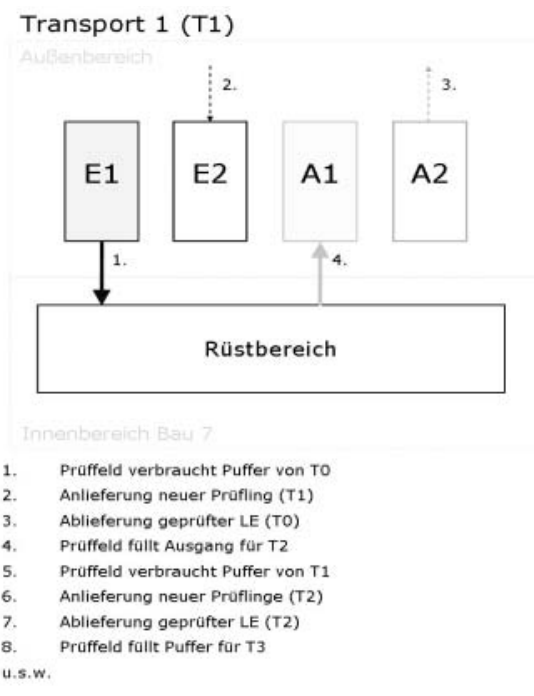

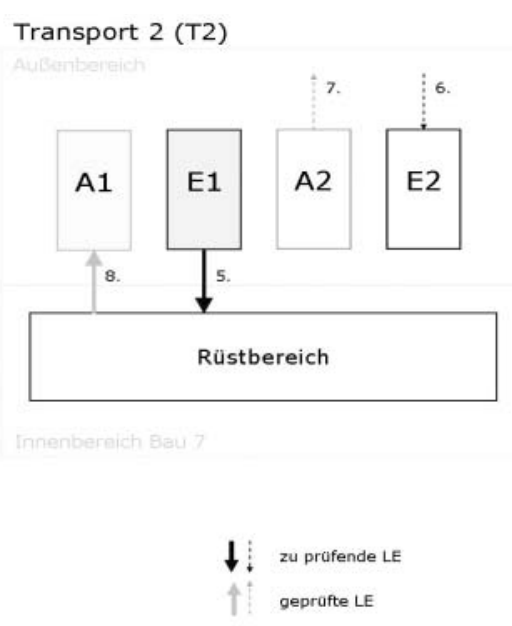

Abb. 7: Prinzipskizze der Variante 4

\section{Systemkosten}

In der Kostenbilanz (Tabelle 4) schlagen sich vor allem die geringen Personalkosten positiv nieder. Trotz relativ hoher Investitions- und Abschreibungskosten fallen die Stückkosten im Verhältnis zu den anderen Planungsvarianten extrem niedrig aus.

\section{Prozessbeurteilung}

Absolute Überlegenheit zeigen die Pufferanhänger mit dem minimalen Handlingaufwand. Vorteile:

- Umbau am Gebäude nicht zwingend erforderlich

- geringer Handlingsaufwand, kurze Prozesszeiten 


\begin{tabular}{|c|c|c|c|}
\hline & Menge Einheit & Preis/Einheit [C] & Kosten [C] \\
\hline Investitionskosten: & & & 294.300 \\
\hline $\begin{array}{l}\text { Frontgabelstapler } \\
\text { Fra }\end{array}$ & 1 Stück & 25.000 & 25.000 \\
\hline Mehraufwand für 2-Ebenen-LKW & 4 Stück & 25.000 & 100.000 \\
\hline Vordach & $350 \mathrm{~m}^{2}$ & 250 & 87.500 \\
\hline Transponder & 250 Stück & 10 & 2.500 \\
\hline Schreib/Lesegeräte mobil & 15 Stück & 3.500 & 52.500 \\
\hline Schreib/Lesegeräte stationär & 14 Stück & 1.200 & 16.800 \\
\hline DV-Technik & 1 Stück & 10.000 & 10.000 \\
\hline \multicolumn{3}{|l|}{ einmalige Gemeinkosten: } & 500 \\
\hline \multicolumn{3}{|l|}{ Baumaßnahmen (Stell platzmakierungen) } & 500 \\
\hline \multirow{5}{*}{$\begin{array}{l}\text { la ufende Kosten pro Jahr: } \\
\text { Personal (indrekte MA) } \\
\text { Abschreibung } \\
\text { kalkulatorische Zinsen } \\
\text { Betriebskosten }\end{array}$} & & & 159.353 \\
\hline & 2 MA p.a. & 52.000 & 104.000 \\
\hline & & & 24.275 \\
\hline & & & $\begin{array}{l}11.036 \\
20.042\end{array}$ \\
\hline & & $\begin{array}{c}\text { Kosten/LE } \\
\text { (bei } 42500 \text { LE/Jahr) }\end{array}$ & $\mathbf{3 , 7 5}$ \\
\hline
\end{tabular}

Tab. 4: Kostenkalkulation für die Variante 4

Nachteile:

- mittlerer Flächenbedarf

- hohes Investitionsvolumen

- eingeschränkte Transparenz und Prozesssicherheit

- störanfällig

\section{Variantenvergleich}

\subsection{Nutzwertanalyse}

Als Entscheidungshilfe sollen die im vorangegangenen Kapitel ausgearbeiteten Varianten nun nach dem Nutzwertverfahren bewertet werden. Dazu wird eine Benotung anhand unterschiedlich gewichteter Kriterien durchgefuihrt. Diese Methode bietet sich besonders für einen nachvollziehbaren Vergleich an, der vorrangig auf der Basis qualitativer Merkmale beruht. Bei einer Nutzwertanalyse wird in fuinf Schritten vorgegangen:

(1) Zunächst werden die Bewertungskriterien definiert, wobei sich die Auswahl nach der Aussagekraft über die Zielfuihrung der einzelnen Varianten richtet.

(2) Jedes Kriterium fließt mit einer bestimmten Wertigkeit in die nachfolgende Nutzwertanalyse ein. Die Wertigkeit entspricht der relativen Bedeutung für die angestrebte Zielerfiillung. Um die Kriterien zu klassifizieren, werden sie einander gegenübergestellt. Im direkten Vergleich wird ihnen eine Wichtigkeit bezüglich der jeweils anderen Kriterien zugeordnet (,wichtiger“, „gleichwertig“ oder „,weniger signifikant"). Tabelle 5 zeigt die aufgrund ihrer besonderen Relevanz für die untersuchten Systemlösungen gewählten Kriterien und ihre Klassifikation.

(3) Anschließend werden die einzelnen Systeme in Hinsicht auf die Erfuillung der verschiedenen Kriterien beurteilt, indem sie für jedes Kriterium benotet werden. Die Skala reicht hier von 5 „sehr gut“ bis 1 „sehr schlecht“. Diese Einschätzung entspricht im Großen und Ganzen dem Stärken-Schwächen-Profil der jeweiligen Variante.

(4) Die relative Bewertung einer Variante bezogen auf ein Kriterium ergibt sich, wenn man absolute Benotung und Gewichtung multipliziert. Spaltenweise werden diese gewichteten Werte (Teilnutzen) zu einem Gesamtergebnis addiert. Gemäß den ermittelten Nutzwerten wird eine Rangfolge gebildet. Tabelle 6 verdeutlicht die Resultate dieses Schrittes.

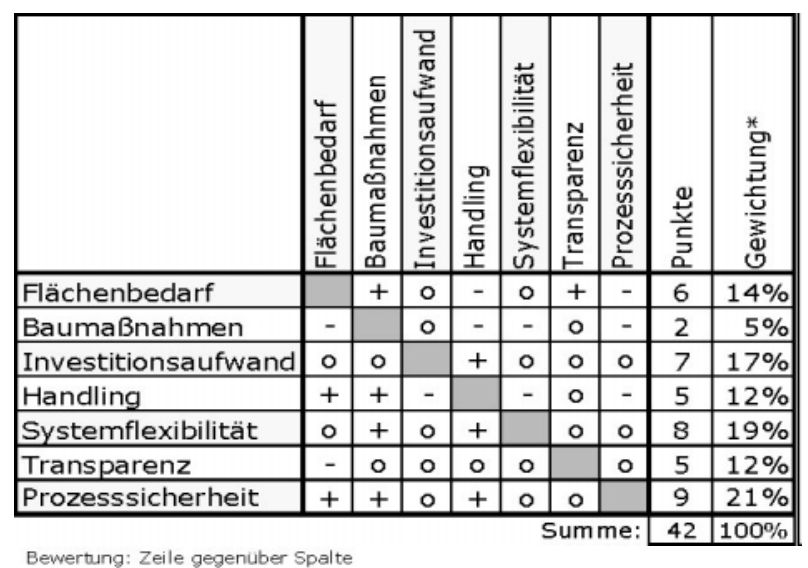

Bevertung: Zeile gegenuber Spalte

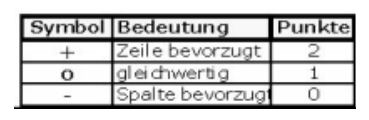

Tab. 5: Präferenzmatrix zur Ermittlung der Kriteriengewichtung (* Gewichtung = prozentualer Anteil an der Summe aller Punkte)

\begin{tabular}{|c|c|c|c|c|c|c|c|c|c|}
\hline \multirow{2}{*}{\begin{tabular}{|l}
$\begin{array}{l}\text { Entscheidungs- } \\
\text { kriterium }\end{array}$ \\
Fochenbedarf
\end{tabular}} & $\begin{array}{c}\text { Gevichtungs- } \\
\text { faktor }\end{array}$ & \multicolumn{2}{|c|}{\begin{tabular}{c|} 
Variante 1 \\
"Schwerlastregal" \\
Note gewichtet
\end{tabular}} & \multicolumn{2}{|c|}{\begin{tabular}{c|} 
Variante 2 \\
"Fahrgestelle" \\
Note gewditer
\end{tabular}} & \multicolumn{2}{|c|}{\begin{tabular}{|c|} 
Variante 3 \\
"Tragkettenförderer" \\
Noevindte.
\end{tabular}} & \multicolumn{2}{|c|}{$\begin{array}{l}\text { Variante } 4 \\
\text { "LKW-Anhänger" } \\
\text { Note gewnchte }\end{array}$} \\
\hline & $14 \%$ & 4 & 0,6 & 1 & 0,1 & 5 & 0,7 & 3 & 0,4 \\
\hline Baumasinthmen & $5 \%$ & 4 & 0,2 & 4 & 0,2 & 1 & 0,1 & 5 & 0,3 \\
\hline Investiogsaufyend & $17 \%$ & 5 & 0,9 & 4 & 0,7 & 2 & 0,3 & 3 & 0,5 \\
\hline Handing & $12 \%$ & 3 & 0,4 & 2 & 0,2 & 3 & 0,4 & 5 & 0,6 \\
\hline Systemfexibilitat & $19 \%$ & 4 & 0,8 & 4 & 0,8 & 1 & 0,2 & 3 & 0,6 \\
\hline Transparenz & $12 \%$ & 4 & 0,5 & 3 & 0,4 & 4 & 0,5 & 3 & 0,4 \\
\hline Frozessichemait & $21 \%$ & 5 & 1,1 & 3 & 0,6 & 4 & 0,8 & 3 & 0,6 \\
\hline Summe: & $100 \%$ & & 4,3 & & 3,0 & & 3,0 & & 3,3 \\
\hline & Ran & & $\overline{1}$ & & 3 & & 3 & & 2 \\
\hline
\end{tabular}

Tab. 6: Nutzwertanalyse

(5) Zum Schluss erfolgt die Aufbereitung der Ergebnisse: In der Rangliste fuihrt Variante 1 mit großem Abstand. Das Schwerlastregal wird in fast allen Punkten am besten benotet. Diese relativ einfache Systemlösung besticht durch Flexibilität, hohe Prozesssicherheit und geringen Investitionsbedarf. Größter Mangel ist der Handlingaufwand, bei dem die LKW-Anhänger am besten eingeschätzt werden. Ansonsten wird Variante 4 eher mittelmäßig gewertet. Variante 2 und 3 erreichen trotz unterschiedlich bewerteter Teilnutzen den gleichen Gesamt-Nutzwert.

Zur Überprüfung der Ergebnisse in Tabelle 6 sowie zur Einschränkung ihres subjektiven Charakters wurde anknüpfend an die Nutzwertanalyse eine Sensitivitätsanalyse mit beliebig veränderten Gewichten der einzelnen Zielkriterien durchgefuihrt.

\subsection{Kostenvergleichsrechnung}

Um die finanziellen Gesichtspunkte gleichermaßen zu berücksichtigen, soll neben der Nutzwertanalyse eine Kostenvergleichsrechnung zur Auswahl einer Vorzugsvariante herangezogen werden. Vergleichsparameter ist hier der fuir die einzelnen Varianten ermittelte Preis pro Ladeeinheit. Aus Tabelle 7 geht hervor, dass Variante 4 dabei am besten abschneidet.

\subsection{Kosten-Nutzen-Analyse}

Die beiden Vergleichsverfahren Nutzwertanalyse und Kostenvergleichsrechnung ergeben zwei unterschied- 


\begin{tabular}{|c|c|c|c|c|}
\hline Variante 1 & Variante 2 & Variante 3 & Variante 4 \\
"Schwerlastregal" & "Fahrgestelle" & "Tragkettenförderer" & "LKW-Anhänger" \\
\cline { 2 - 5 } & 5,71 & 6,19 & 6,76 & 3,75 \\
\hline
\end{tabular}

Tab. 7: Stückkostenvergleich

liche Lösungsempfehlungen. Da es außerdem nicht sinnvoll ist, Nutzwert und Kosten isoliert voneinander zu betrachten, wird hier als Kosten-Nutzen-Analyse fuir die Ermittlung einer zu favorisierenden Variante der „ordinale“ Nutzwert ins Verhältnis zu den Kosten gesetzt. Das Ergebnis dieser Vorgehensweise gibt folgende Grafik wieder:

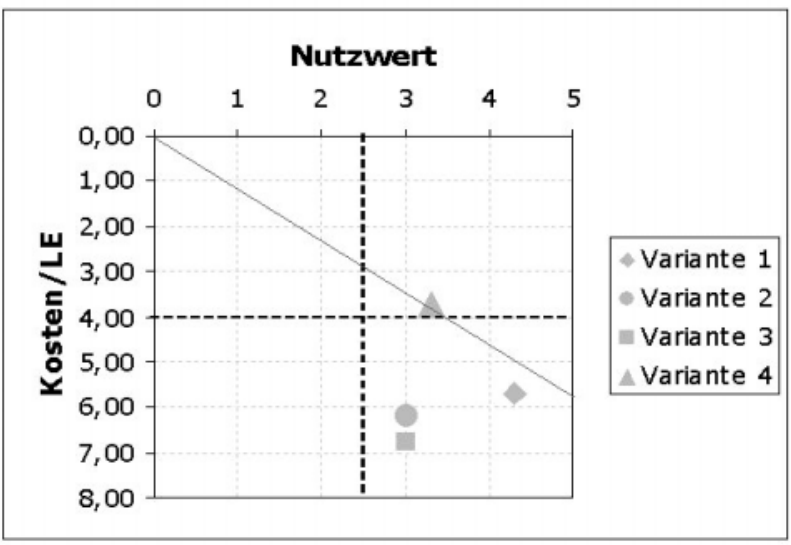

Abb. 8: Kosten-Nutzen-Verhältnis

Auf der X-Achse ist der Nutzwert entsprechend Tabelle 6 abgetragen, die Y-Achse stellt die Kosten aus Tabelle 7 dar. Zu präferieren sind dabei die Varianten, die sich in dem rechten oberen Viertel der Matrix befinden - also einen hohen Nutzwert bei geringen Stuickkosten aufweisen. Im Gegensatz dazu bieten die Lösungen im linken unteren Viertel das schlechteste Verhältnis zwischen Nutzen und Kosten. Demzufolge bietet die Lösung 4 offensichtlich die beste Kosten-Nutzen-Relation aller hier untersuchten Varianten. Die Gerade, die durch Variante 4 verläuft, kennzeichnet dabei alle möglichen Kombinationen mit dem gleichen Kosten-Nutzen-Verhältnis. Alle Punkte unterhalb der Geraden sind schlechter, alle dariiber besser gestellt. Variante 1 nähert sich der Geraden am weitesten (von unten) und stellt somit das zweitbeste Kosten-Nutzen-Verhältnis.

\section{Empfehlung}

Wie die Kosten-Nutzen-Analyse gezeigt hat, ist die Variante 4 mit den LKW-Anhängern im Gesamtvergleich zu favorisieren. Die Vorzüge dieser Lösung liegen in dem minimalen Handlingaufwand sowie der damit verbundenen Konsequenz eines geringen Personalaufwandes. Dadurch wiederum können die Prozesskosten auf dem niedrigsten Niveau gehalten werden. Zwar geht die Variante „Schwerlastregal“ im qualitativen Vergleich als klarer Sieger hervor, gegenüber den LKW-Anhängern offenbart sie jedoch erhebliche Kostennachteile, die aus dem höheren Personalbedarf resultieren. Infolgedessen stellt sich das Kosten-Nutzen-Verhältnis der Variante 1 schlechter dar als jenes der Variante 4. Fahrgestelle und Tragkettenförderer konnten sich dagegen weder im Kostenvergleich noch in der Nutzbewertung durchsetzen.
Als Ergebnis dieser Arbeit ist daher die Variante 4 für die weiterführende Feinplanung zu bevorzugen. Im Anschluss an die vorliegende Untersuchung wird die Entscheidung uiber eine umzusetzende Systemlösung getroffen werden und die Feinplanung der entsprechenden Logistikprozesse beginnen. Deren Aufgaben werden hauptsächlich darin bestehen, Realisierungsstufen zu planen, Schnittstellen zu überprüfen, Genehmigungsverfahren einzuleiten sowie später die Ausschreibungsphase zu betreuen. Diese umfasst die Lastenhefterstellung und den Vergleich von Angeboten verschiedener Hersteller. Nachdem letztlich ein Systemanbieter ausgewählt wurde, wird die Auftragsvergabe mit den zugehörigen Bestellvorgängen erfolgen.

\section{Literatur}

Ihme, Joachim: Logistik im Fahrzeugbau; Manz Schulbuch, Wien 2000

Jünemann, Reinhardt \& Schmidt, Thorsten: Materialflusssysteme. Systemtechnische Grundlagen; 2. Auflage, SpringerVerlag, Berlin [u. a.] 2000

Jünemann, Reinhardt \& Beyer, Andreas: Steuerung von Materialfluß- und Logistiksystemen; 2. Auflage, Springer-Verlag, Berlin [u. a.] 1998

Kluck, Dieter: Materialwirtschaft und Logistik; 2. Auflage, Schäffer-Poeschel Verlag, Stuttgart 2002

Martin, Heinrich: Transport- und Lagerlogistik - Planung, Aufbau und Steuerung von Transport- und Lagersystemen; 3. Auflage, Vieweg-Verlag, Braunschweig/Wiesbaden 2000

\section{Autoren}

Dipl.-Wirtschaftsing. (FH) Bianca Pellegrini

Prof. Dr.-Ing. Herbert Sonntag

Technische Fachhochschule Wildau

Fachbereich Ingenieurwesen/Wirtschaftsingenieurwesen Logistik

Telefon +493375 508-924

E-Mail: hsonntag@igw.tfh-wildau.de 